\title{
XB130 enhances invasion and migration of human colorectal cancer cells by promoting epithelial-mesenchymal transition
}

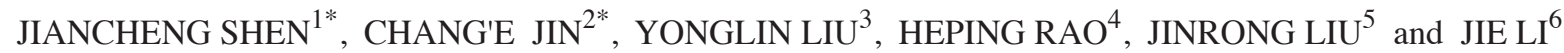 \\ ${ }^{1}$ Clinical Laboratory, Shaoxing Hospital of Traditional Chinese Medicine, Shaoxing, Zhejiang 312499; \\ ${ }^{2}$ Intensive Care Unit, Laigang Hospital Affiliated to Taishan Medical University, Laiwu, Shandong 272009; \\ ${ }^{3}$ Clinical Laboratory, Zhejiang Provincial Hospital of Traditional Chinese Medicine, Hangzhou, Zhejiang 310002; \\ ${ }^{4}$ Department of Nursing, School of Medicine, Quzhou College of Technology, Quzhou, Zhejiang 324000; \\ ${ }^{5}$ Department of Child Healthcare; ${ }^{6}$ Department of Infectious Disease, Second Affiliated Hospital \\ of Wenzhou Medical University, Wenzhou, Zhejiang 325027, P.R. China
}

Received October 16, 2016; Accepted June 8, 2017

DOI: $10.3892 / \mathrm{mmr} .2017 .7279$

\begin{abstract}
The expression of XB130 is associated with invasion and migration of many tumor cells, but its roles in human colorectal cancer (CRC) remains unknown. To investigate this, protein expression levels of XB130 in numerous human CRC cell lines were compared with a normal colorectal mucosa cell line by western blotting. Knockdown of XB130 using small interfering (si)RNA was performed to assess the effects on cell invasion and migration in a Transwell assay and a scratch test. Western blotting was also used to quantify the levels of proteins associated with epithelial-mesenchymal transition (EMT), including E-cadherin, vimentin, phosphorylated (p)-protein kinase B (AKT), p-forkhead homeobox type O 3a (FOXO3a) and zinc finger E-box-binding homeobox 1 (ZEB-1). The relative expression of XB130 protein was significantly higher in CRC cells compared with control cells $(\mathrm{P}<0.01)$. Knockdown of XB130 using siRNA significantly decreased the invasive and migratory responses of $\mathrm{CRC}$ cells $(\mathrm{P}<0.01)$. In addition, levels of E-cadherin were increased, while vimentin, $\mathrm{p}$-AKT, p-FOXO3a and ZEB-1 were decreased $(\mathrm{P}<0.01)$. In conclusion, the present study demonstrated that the expression of XB130 is elevated in CRC cells. Loss of XB130 was associated with decreased invasion and migration of CRC cells, possibly as a
\end{abstract}

Correspondence to: Dr Jie Li, Department of Infectious Disease, Second Affiliated Hospital of Wenzhou Medical University, 109 West Xueyuan Road, Wenzhou, Zhejiang 325027, P.R. China E-mail: jaly1111@126.com

Dr Jinrong Liu, Department of Child Healthcare, Second Affiliated Hospital of Wenzhou Medical University, 109 West Xueyuan Road, Wenzhou, Zhejiang 325027, P.R. China

E-mail: liujinrong126@163.com

*Contributed equally

Key words: colorectal cancer, XB130, cell invasion, cell migration, epithelial-mesenchymal transition result of EMT inhibition. Thus, upregulation of XB130 may underlie some of the tumorigenic events observed in human CRCs. XB130 may be a promising target for CRC therapy in humans; further mechanistic studies exploring the function of $\mathrm{XB130}$ in CRC cells are warranted.

\section{Introduction}

Colorectal cancer (CRC) is one of the most common malignancies in the world, accounting for $10 \%$ of all cancers $(1,2)$. The mortality rate for CRC is the fourth highest globally $(1,2)$. CRC cells have a strong tendency to migrate and invade healthy tissues. As a result, treatments in patients with advanced CRC often fail due to extensive infiltration or distant metastasis. Therefore, inhibiting the invasion and migration of CRC cells would significantly improve therapeutic treatments for CRC, and the overall prognosis of patients (3-5). However, the regulation of invasion and migration of CRC cells likely involves the activities of many genes. In particular, the signaling molecules and pathways at work are likely complex, and have not yet been sufficiently elucidated.

Epithelial-mesenchymal transition (EMT) of tumor cells occurs when cells possessing epithelial characteristics take on a mesenchymal phenotype with respect to their morphology, and subsequently display enhanced proliferation, invasion, migration and drug resistance (6). E-cadherin is typically expressed in cells displaying an epithelial phenotype, and vimentin is a marker associated with mesenchymal cell phenotypes (7). EMT of tumor cells is controlled in a variety of ways, such as through non-coding RNAs, differential splicing, transcriptional control and post-transcriptional regulation (8). Zinc finger E-box-binding homeobox 1 (ZEB-1) is considered the most important transcription factor required for inducing EMT, and its expression is modulated by the aforementioned processes (9). ZEB-1 binds to E2 box, which serves as an E-cadherin gene promoter, and inhibits transcription of E-cadherin (6). The phosphorylated (p) -protein kinase B (AKT) signaling pathway can phosphorylate forkhead homeobox type $\mathrm{O}$ (FOXO)3a, which is a member of the FOXO family of transcriptional factors (10). FOXO3a can regulate the activity of the $\beta$-catenin/T-cell factor 
(TCF) transcriptional complex. Phosphatidyl-inositol-3-kinase (PI3K)/AKT-mediated FOXO3a phosphorylation may lead to its export from the nucleus (11). p-AKT also inhibits the Wnt signaling pathway, which normally promotes the expression of ZEB-1 (12).

XB130 protein, also known as actin filament associated protein 1L2, is a novel adaptor protein which mediates RET/PTC chromosome rearrangement-related thyroid cancer cell proliferation and survival through the PI3K/AKT signaling pathway (6). Its expression is elevated in thyroid, gastric and esophageal cancers, and is implicated in processes such as survival and proliferation of tumor cells and the expression of some tumor suppressive microRNAs $(13,14)$. Previous studies have also provided functional evidence that XB130 protein is involved in the regulation of cell invasion and migration in gastric cancer, breast cancer, osteosarcoma, and other malignant tumors $(15,16)$. Furthermore, inhibiting the expression of XB130 significantly reduces the invasion and migration abilities of these tumor cells $(15,17)$. XB130 is implicated in the development of some cancers; however, its roles in CRC are not clear. In the present study, RNA interference was used to inhibit expression of XB130 in CRC cell lines to assess the effects on tumor cell invasion and migration. The results demonstrated that XB130 is highly expressed in CRC cells, and XB130 knockdown significantly reduced the invasive and migratory abilities of CRC cells, potentially as a result of a reduction in EMT. Taken together, these results suggested that XB130 might increase the tendency of CRC cells to migrate and invade other tissues through activation of EMT.

\section{Materials and methods}

Cell lines and reagents. SW620, LoVo and SW480 human CRC cell lines and FHC colorectal mucosa cells were purchased from the Chinese Academy of Sciences Typical Culture Preservation Committee Cell Bank (Shanghai, China). SW620 and LoVo cells were cultured in RPMI1640 medium (Thermo Fisher Scientific, Inc. Waltham, MA, USA), and SW480 and FHC cells were cultured in Dulbecco's modified Eagle's medium (Thermo Fisher Scientific, Inc.) supplemented with $10 \%$ fetal bovine serum (Thermo Fisher Scientific, Inc.) and $1 \%$ penicillin-streptomycin (Thermo Fisher Scientific, Inc.). All cells were maintained in a humidified $5 \% \mathrm{CO}_{2}$ incubator at $37^{\circ} \mathrm{C}$. Rabbit anti-XB130 (cat no. ab106433), anti-E-cadherin (cat no. ab40772), anti-Vimentin (cat no. ab92547), anti-phosphorylated (p)-AKT1(S473; cat no. ab81283), anti-p-FOXO3A (S253; cat no. ab47285), anti-ZEB-1 (cat no. ab203829) and anti-GAPDH (cat no. ab9485) primary antibodies, in addition to a horseradish peroxidase-conjugated goat anti-rabbit immunoglobulin G secondary antibody (cat no. ab205718), were obtained from Abcam (Shanghai, China). Specific XB130-small interfering (si)RNA containing a pool of 3-5 target-specific 19-25 nucleotide sequences (cat. no. sc-90824) and a negative control (NC)-siRNA (cat. no. sc-37007) were produced by Santa Cruz (Dallas, TX, USA). Lipofectamine-2000, TRIzol reagent and Opti-MEM transfection medium were purchased from Thermo Fisher Scientific, Inc. Matrigel was produced by BD Biosciences (San Jose, CA, USA). Prime Script Reagent RT and SYBR Green kits were obtained from Takara Biotechnology Co., Ltd. (Dalian, China).

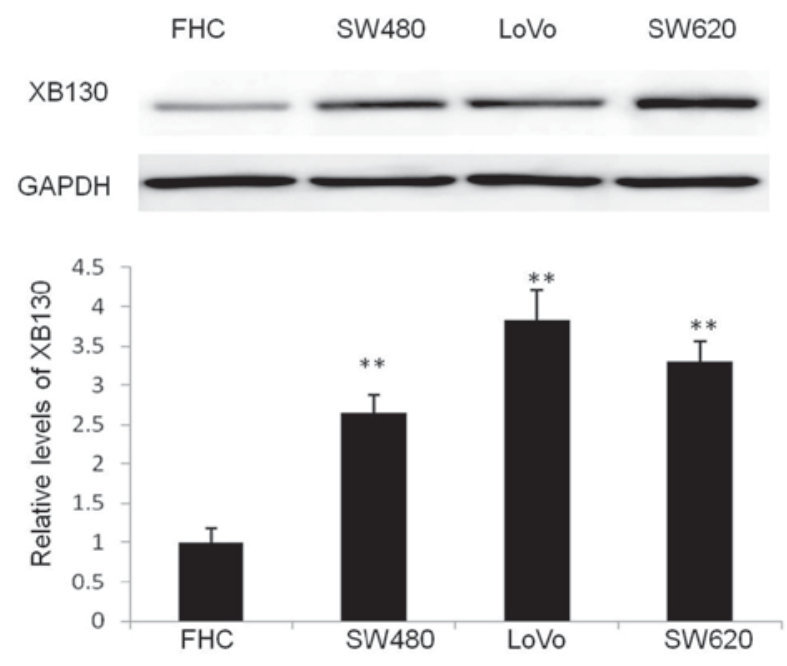

Figure 1. Quantification of XB130 protein expression levels in SW620, LoVo and SW480 colorectal cancer cell lines compared with FHC control cells. GAPDH served as an internal reference. Data are presented as the mean \pm standard deviation. ${ }^{* *} \mathrm{P}<0.01$ vs. FHC.

Knockdown of XB130 using siRNA. Cells ( $2 \times 10^{5}$ cells/well) were seeded into 6 -well plates and cultured to $50 \%$ confluence. The media was then discarded and changed to serum-free media for further culturing. Opti-MEM was used to dilute $100 \mathrm{pmol}$ of XB130-siRNA or NC-siRNA in $250 \mu \mathrm{l} / \mathrm{well}$, and cells were then incubated for $5 \mathrm{~min}$ at $37^{\circ} \mathrm{C}$. In a separate tube, Lipofectamine $2000(5 \mu \mathrm{l})$ was diluted with Opti-MEM and added to each vial containing diluted siRNA for $15 \mathrm{~min}$ at $37^{\circ} \mathrm{C}$. The serum-free media in 6-well plates was discarded and replaced with the transfected solution with an additional $2 \mathrm{ml} /$ well Opti-MEM. Cultures were incubated for $6 \mathrm{~h}$ at $37^{\circ} \mathrm{C}$ under $5 \% \mathrm{CO}_{2}$ before replacing the media with fresh normal media. XB130 mRNA expression levels were assayed by reverse transcription-quantitative polymerase chain reaction (RT-qPCR) after $24 \mathrm{~h}$, and XB130 protein expression levels were quantified by western blotting after $48 \mathrm{~h}$.

Transwell invasion assay. Matrigel was thawed for $24 \mathrm{~h}$ at $4^{\circ} \mathrm{C}$. Transwell inserts with an $8-\mu \mathrm{m}$ pore size were coated with $100 \mu \mathrm{l}$ diluted Matrigel (1:8 dilution with serum-free media) for $30 \mathrm{~min}$ at $37^{\circ} \mathrm{C}$. Serum-free media $(500 \mu \mathrm{l})$ was added to the lower chamber, which was then incubated for $30 \mathrm{~min}$ at $37^{\circ} \mathrm{C}$. The SW620 cells transfected with XB130-siRNA, NC-siRNA or a blank control were counted under an Olympus BX63 microscope (Olympus Corporation, Tokyo, Japan), and serum-free medium was subsequently added at a concentration of $2 \times 10^{5}$ cells $/ 200 \mu \mathrm{l}$. The cell suspension was applied to the upper chamber, and $10 \%$ FBS media was added to the lower chamber. Following incubation, cells in the upper chamber were removed with cotton swabs and cells that traversed the Matrigel to the lower surface of the insert were fixed with $10 \%$ formalin containing $0.1 \%$ crystal violet for $30 \mathrm{~min}$ at room temperature. After washing with PBS several times, the stained cells were imaged under a microscope (Olympus Corporation) and decolorized using 33\% acetic acid. The OD values for the eluents were measured at $570 \mathrm{~nm}$ with a spectrophotometer (UNICO, Suite E Dayton, NJ, USA), and relative cell numbers were quantified. 
A
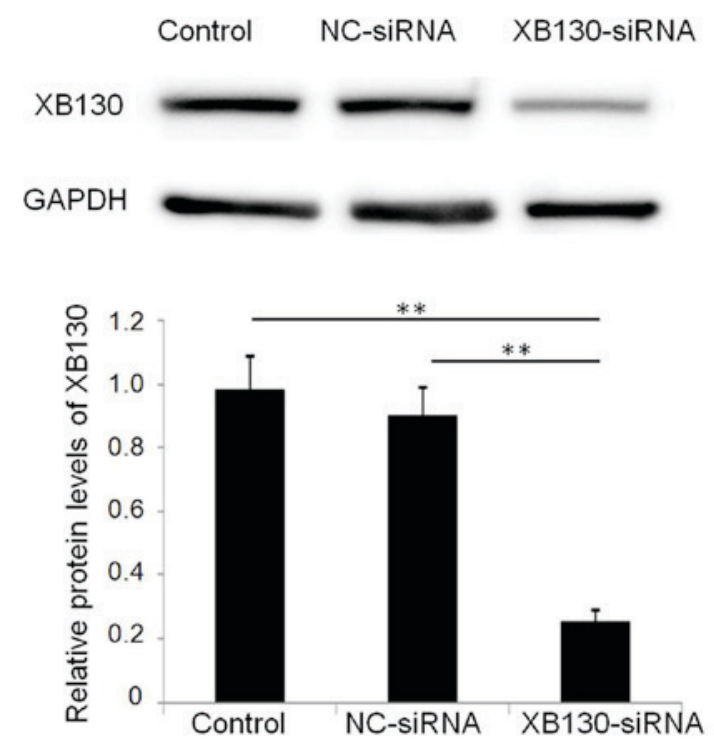

B

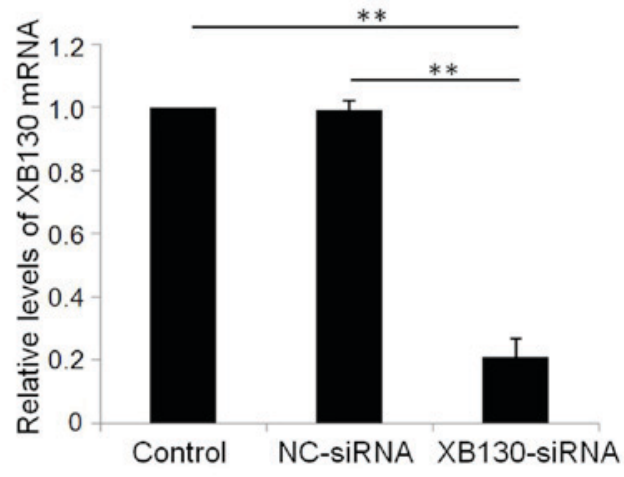

Figure 2. Expression of XB130 after siRNA transfection in SW620 cells. XB130 (A) protein and (B) mRNA expression levels, as assessed by western blotting and reverse transcription-quantitative polymerase chain reaction, respectively. Data are presented as the mean \pm standard deviation. ${ }^{* *} \mathrm{P}<0.01$. $\mathrm{NC}$, negative control; siRNA, small interfering RNA.

Scratch assay. Cells transfected with XB130-siRNA, NC-siRNA or blank controls were harvested. Approximately $1-5 \times 10^{5}$ cells/well were subsequently seeded into 6 -well plates and grown to $90 \%$ confluence overnight. The confluent monolayer was scraped with a pipette tip, creating a scratch in each well. The scraped cells were discarded by washing three times with PBS. Serum-free media was added, and cells were incubated at $37^{\circ} \mathrm{C}$ under $5 \% \mathrm{CO}_{2}$. Cells were imaged at different time points $(0,6,12$ and $24 \mathrm{~h})$, and the relative scratch area was determined by dividing the area devoid of cells at 24 h by the area devoid of cells at $0 \mathrm{~h}$ using Olympus Stream Image Analysis software version 1.0 (Olympus Corporation).

Western blotting. Samples containing $1 \times 10^{6}$ cells were lysed in $200 \mu \mathrm{l}$ cell lysis buffer (Cell Signaling Technology, Beverly, MA, USA) for $30 \mathrm{~min}$ at $4^{\circ} \mathrm{C}$, and then centrifuged at $8,000 \mathrm{x} \mathrm{g}$ for $10 \mathrm{~min}$ at $4^{\circ} \mathrm{C}$. Protein concentrations for each sample were determined using a Bicinchoninic Acid protein assay kit (Pierce; Thermo Fisher Scientific, Inc.) with 10\% bovine serum albumin (Sigma-Aldrich; Merck KGaA, Darmstadt, Germany) as a standard.

Western blotting was performed as previously described $(18,19)$. Briefly, $10 \mu \mathrm{g}$ protein was separated by 4-12\% SDS-PAGE, followed by transfer to an Immobilon polyvinylidene difluoride membrane (EMD Millipore, Billerica, MA, USA). After blocking with $5 \%$ non-fat dry milk in $50 \mathrm{mM}$ Tris- $\mathrm{HCl}$ (pH 7.6), $150 \mathrm{mM} \mathrm{NaCl}$ and $0.1 \%$ Tween-20 (TBST), anti-XB130, anti-E-cadherin, anti-vimentin, anti-AKT1 (phospho S473), anti-FOXO3A (phospho S253), anti-ZEB-1 and anti-GAPDH primary antibodies were diluted at 1:1,000-2,000 in TBST, with the final concentration of $1-2 \mu \mathrm{g} / \mathrm{ml}$ and were incubated with the blots overnight at $4^{\circ} \mathrm{C}$. After washing and incubating with a horseradish peroxidase-conjugated secondary antibody with a dilution of $1: 1,000$ for $2 \mathrm{~h}$ at $4^{\circ} \mathrm{C}$, the membrane was developed with an enhanced chemiluminescent reagent (EMD Millipore) and imaged using Image Quant LAS 500 (GE Healthcare, Fairfield, CT, USA). Image Quant TL version 8.1 software was used for densitometry (GE Healthcare). GAPDH was used as an internal reference.

$R N A$ isolation and RT-qPCR. Total RNA was extracted with TRIzol (Thermo Fisher Scientific, Inc.) and reverse transcribed into cDNA in a total volume of $20 \mu \mathrm{l}$ using a PrimeScript ${ }^{\mathrm{TM}}$ II High Fidelity RT-PCR kit (Takara Biotechnology Co., Ltd.). qPCR was performed using a DNA Engine Chromo 4 Real-time Quantitative PCR (qPCR) system (Bio-Rad Laboratories, Inc., Hercules, CA, USA) and a SYBR Green kit (Takara Biotechnology, Co., Ltd.). GAPDH was used as an internal control. Primer sequences for XB130 were 5'-AGCACAGCA CTGGTGAAGAA-3' (forward) and 5'-GTTGCTTGTTGA TGGTCACT-3' (reverse). Primers for $G A P D H$ were 5'-CCA TGTTCGTCATGGGTGTG-3' (forward) and 5'-GGTGCTAAG CAGTTGGTGGTG-3' (reverse). The reaction mix included $10 \mu \mathrm{l}$ SYBR Green Master Mix, $0.2 \mu \mathrm{l}$ each gene-specific forward and reverse primer (10 pmol $/ \mu \mathrm{l}), 2 \mu \mathrm{l} \mathrm{cDNA}$ and $7.8 \mu \mathrm{l}$ $\mathrm{H}_{2} \mathrm{O}$. qPCR was performed for 40 cycles of denaturation at $95^{\circ} \mathrm{C}$ for $45 \mathrm{sec}$, annealing at $62^{\circ} \mathrm{C}$ for $30 \mathrm{sec}$ ) and extension at $72^{\circ} \mathrm{C}$ for $30 \mathrm{sec}$. Double-stranded DNA was measured at $86^{\circ} \mathrm{C}$ after each cycle. The relative expression levels of XB130 and GAPDH were calculated by $2^{\Delta \Delta \mathrm{Cq}}$ method (20). Each experiment was performed in triplicate.

Statistical analysis. Every experiment was repeated three times, and data are presented as the mean \pm standard deviation. Statistical significance was calculated using SPSS v.20 software (SPSS Inc., Chicago, IL, USA). Student's t-test was used to compare two groups, and one-way analysis of variance followed by a post hoc Tukey test was used when comparing 

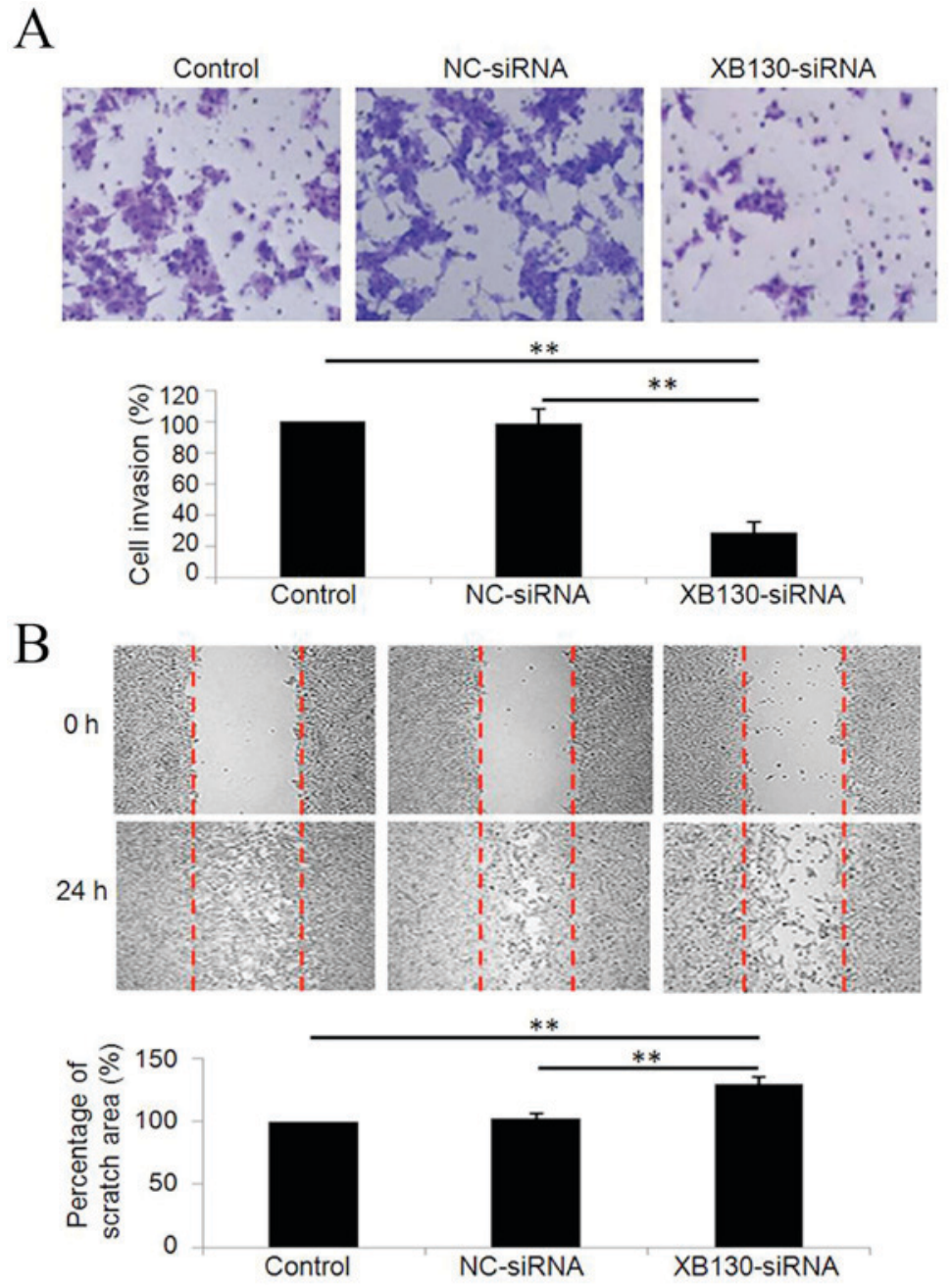

Figure 3. Effect of XB130 siRNA knockdown on the invasion and migration of SW620 cells. (A) Invasion and migration of SW620 cells, assayed by a Transwell test (magnification, $\mathrm{x} 200$ ). (B) Invasion and migration of SW620 cells, assayed by a scratch test (magnification, $\mathrm{x} 100$ ). Data are presented as the mean \pm standard deviation. ${ }^{* * *} \mathrm{P}<0.01$. NC, negative control; siRNA, small interfering RNA.

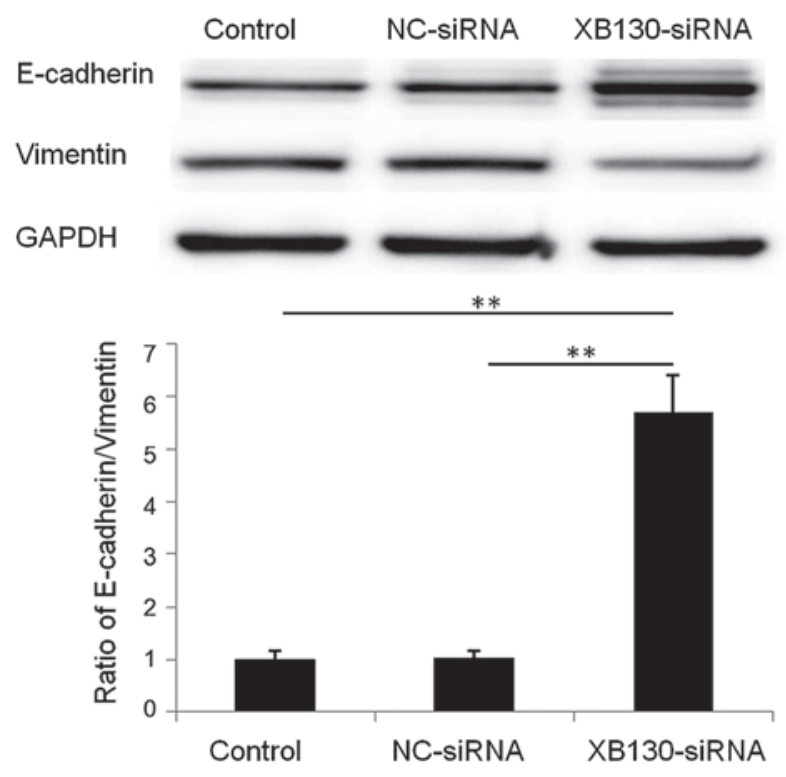

Figure 4. Effects of XB130 knockdown in SW620 cells on the expression of epithelial-mesenchymal transition-associated markers. Western blotting was used to detect the protein expression of E-cadherin and vimentin. GAPDH served as an internal control. Data are presented as the mean \pm standard deviation. ${ }^{* *} \mathrm{P}<0.01$. NC, negative control; siRNA, small interfering RNA. more than three groups. All tests for significance were two-sided, and $\mathrm{P}<0.05$ was considered to indicate a statistically significant difference.

\section{Results}

XB130 expression was significantly elevated in three different CRC cell lines compared with normal colorectal mucosa FHC cells. Protein expression levels of XB130 were normalized with the expression levels of GAPDH in different cell lines when examined by western blotting. Compared with normal colorectal mucosa FHC cells, the relative expression level of XB130 protein was significantly elevated in SW620, LoVo and SW480 human CRC lines $(2.62 \pm 0.13,3.76 \pm 024$, and 3.38 \pm 0.15 , respectively; $\mathrm{P}<0.01$; Fig. 1).

Knockdown of XB130 significantly decreases migration and invasion of SW620 CRC cells. To determine its effects on cell invasion and migration, XB130 expression was knocked down in SW620 cells using siRNA. First, the knockdown efficiency was examined by western blotting. Compared with the relative expression level of XB130 protein in NC-siRNA transfected cells $(0.89 \pm 0.09)$ and the blank control group $(0.92 \pm 0.11)$, 
A

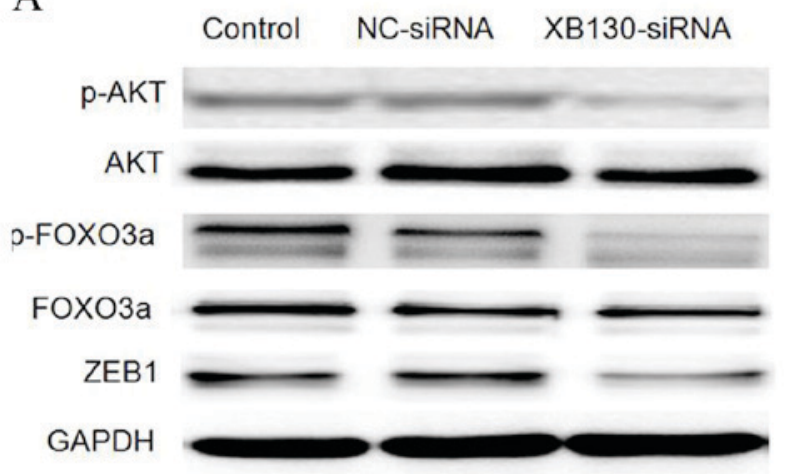

$\mathrm{C}$

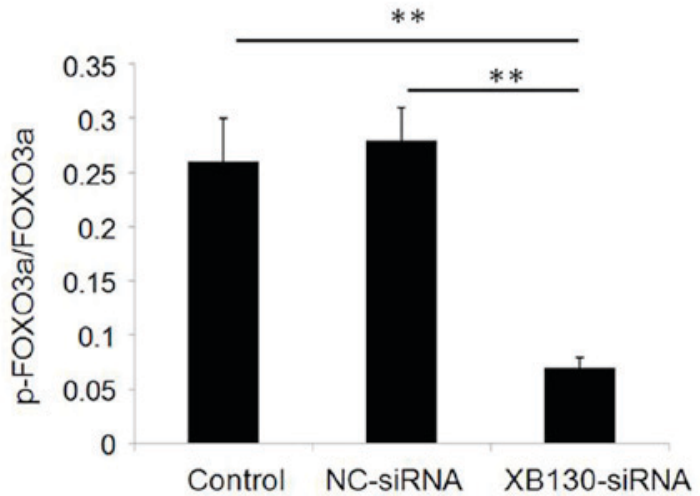

B

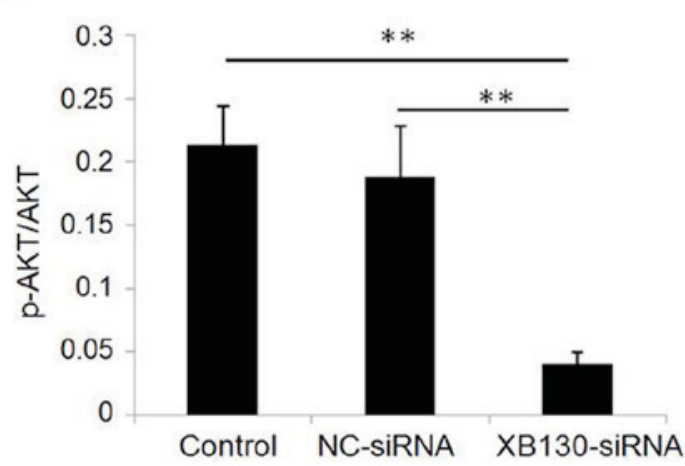

$\mathrm{D}$

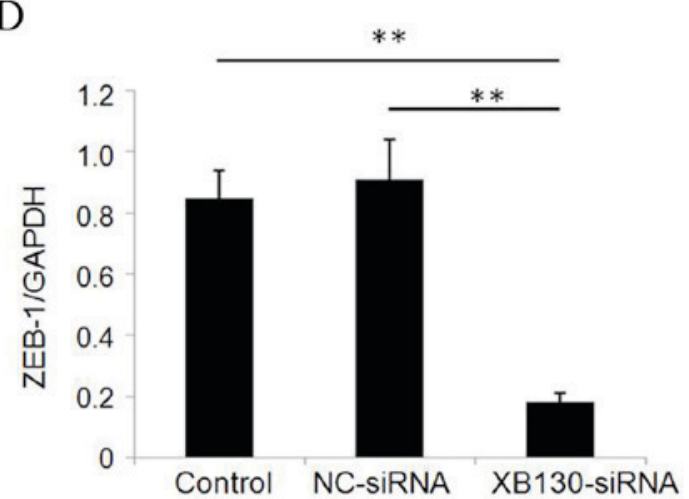

Figure 5. Effect of siRNA knockdown of XB130 on the levels of epithelial-mesenchymal transition-associated transcription factors in SW620 cells. (A) Representative western blot images. Quantification of protein expression levels of (B) p-AKT, AKT, (C) p-FOXO3a, FOXO3a, and (D) ZEB-1 and GAPDH. Data are presented as the mean \pm standard deviation. ${ }^{* *} \mathrm{P}<0.01$. NC, negative control; siRNA, small interfering RNA; AKT, protein kinase B; p, phosphorylated; FOXO3a, forkhead homeobox type O 3a; ZEB-1, zinc finger E-box-binding homeobox 1.

cells transfected with XB130-siRNA $(0.28 \pm 0.04)$ exhibited a significant reduction in $\mathrm{XB1} 30$ ( $\mathrm{P}<0.01$; Fig. 2A). These results were corroborated by RT-qPCR, which demonstrated that $X B 130$ mRNA expression levels were reduced to $0.20 \pm 0.05 \%$ (P<0.01; Fig. 2B).

To test the invasive abilities of cells, a Transwell assay was performed after transfecting SW620 cells with either XB130-siRNA or NC-siRNA. The percentage of cells found in the Transwell chamber were $98.82 \pm 9.24 \%$ for NC-siRNA cells and $28.44 \pm 6.68 \%$ for the XB130-siRNA cells compared with blank control cells $(\mathrm{P}<0.01$, Fig. $3 \mathrm{~A})$. A scratch was also performed to measure cell migration. The scratch area was $102.33 \pm 4.32 \%$ for the NC-siRNA cells and $129.73 \pm 5.92 \%$ for XB130-siRNA cells compared with blank control cells ( $\mathrm{P}<0.01$; Fig. 3B). Thus, these two assays indicated that the XB130-siRNA cells exhibited a statistically significant decrease in both migration and invasion compared with NC-siRNA cells.

XB130 is required for expression of EMT-associated markers in SW620 cells. The expression of the EMT-associated markers E-cadherin and vimentin in SW620 cells was examined by western blotting. The expression levels of E-cadherin relative to GAPDH in SW620 cells of the control, NC-siRNA-transfection and XB130-siRNA transfection groups were $0.23 \pm 0.04$, $0.25 \pm 0.03$ and $0.72 \pm 0.05$, respectively, while the relative levels of vimentin were $0.97 \pm 0.08,1.01 \pm 0.06$, and $0.31 \pm 0.03$, respectively (data not shown). Compared with the NC-siRNA transfection group, the XB130-siRNA cells exhibited markedly increased expression of E-cadherin and significantly decreased expression of vimentin $(\mathrm{P}<0.01$, Fig. 4). Furthermore, the ratio of E-cadherin to vimentin was significantly increased $(\mathrm{P}<0.01$, Fig. 4). No differences in E-cadherin and vimentin expression were identified between the NC-siRNA and control groups.

XB130 knockdown inhibits activation of signaling pathways that facilitate EMT in SW620 cells. Finally, the effects of XB130 knockdown on activation of EMT-associated transcription factors in SW620 cells. Western blotting demonstrated that the proportions of p-AKT to AKT in the control, NC-siRNA cells and XB130-siRNA cells were $0.21 \pm 0.03$, $0.19 \pm 0.04$ and $0.04 \pm 0.01$, respectively $(\mathrm{P}<0.01$, Fig. $5 \mathrm{~A}$ and $\mathrm{B})$. The proportions of p-FOXO3a to FOXO3a were $0.26 \pm 0.04$, $0.28 \pm 0.03$ and $0.07 \pm 0.01$, respectively $(\mathrm{P}<0.01$, Fig. $5 \mathrm{~A}$ and $\mathrm{C})$, and the proportions of ZEB- 1 to GAPDH were $0.85 \pm 0.09$, $0.91 \pm 0.13$ and $0.18 \pm 0.03$, respectively $(\mathrm{P}<0.01$, Fig. $5 \mathrm{~A}$ and D). Compared with the NC-siRNA cells, the relative levels of p-AKT, p-FOXO3a and ZEB-1 were significantly decreased in XB130-siRNA transfected cells.

\section{Discussion}

The biological activity of CRC is complex, and includes frequent relapses, metastasis and drug resistance to chemotherapy (21-23). Therefore, it is imperative to determine 
the molecular mechanisms underlying the occurrence and progression of $\mathrm{CRC}$, in order for earlier and more effective diagnostic methods to be developed, in addition to more robust therapeutic strategies. The present study examined the potential roles for XB130 in the etiology of CRC. In vitro knockdown experiments supported a role for $\mathrm{XB} 130$ in CRC cell invasion and migration, potentially via inhibition of EMT.

The XB130 gene is located at chromosomal position 10q25.3. The XB130 protein contains 818 amino acids with a total molecular weight of $130 \mathrm{kDa}$. Its expression is elevated in many kinds of malignant tumors, and it strongly enhances tumor cell invasion and migration (13-17). Therefore, XB130 might also contribute to the occurrence and development of CRC. The present study explored the potential roles for $X B 130$ in the invasive and migratory behaviors of CRC tumor cell lines. It was demonstrated that the expression of XB130 protein in SW620, LoVo and SW480 CRC cell lines was significantly higher than in the FHC normal colorectal epithelial cell line. In addition, after silencing XB130 in SW620 cells for $48 \mathrm{~h}$ using siRNA, cell invasion and migration were markedly inhibited.

$\mathrm{XB130,}$ which is highly expressed in gastric cancer, can induce EMT-like responses in tumor cells (7). In order to explore the mechanisms underlying the invasive and migratory behaviors observed in CRC cells, the present study examined whether XB130 might affect EMT-associated responses. The results indicated that silencing of XB130 for $48 \mathrm{~h}$ in SW620 $\mathrm{CRC}$ cells caused an increase in the cell membrane protein E-cadherin. Meanwhile, vimentin was significantly downregulated in CRC cells after loss of $X B 130$. This resulted in an overall increase in the ratio of E-cadherin to vimentin, which has been previously observed by Shi et al (7), who demonstrated that knockdown of XB130 in gastric carcinoma caused the same increase in the ratio of E-cadherin to vimentin. Cumulatively, this indicates that EMT may be inhibited after downregulation of $X B 130$, which could explain the reduction in invasion and migration that was observed in CRC cells in the present study.

XB130 contains several tyrosine phosphorylation sites. When phosphorylated, tyrosine can bind the PI3K subunit of p $85 \alpha$ to activate AKT and regulate the cell cycle, apoptosis and other important processes (23). To explore the possibility that XB130 regulates EMT of CRC cells, the levels of p-AKT, p-FOXO3a and ZEB-1 were examined. All three transcription factors levels decreased significantly after knockdown of XB130. Therefore, inhibition of XB130 in CRC cells may lead to a decrease in phosphorylation of AKT. As a result, FOXO3a may not be inactivated by phosphorylation, and it instead might downregulate the activity of the $\beta$-catenin/TCF transcriptional complex. This downregulation of canonical Wnt signaling could result in the target gene $Z E B-1$ not being expressed. Ultimately, this cascade of molecular events might inhibit EMT by decreasing cell invasion and migration in CRC cells (24).

In conclusion, the present study investigated the potential roles for XB130 in promoting CRC cell invasion and migration. These results suggested that the expression of XB130 is significantly elevated in CRC cell lines, and it likely promotes cell behaviors associated with invasion and migration. XB130 may achieve this by promoting regulatory responses that initiate EMT. Therefore, XB130 may be a promising target for $\mathrm{CRC}$ therapy in humans. Further mechanistic studies exploring the function of XB130 in CRC cells are warranted.

\section{Acknowledgements}

The present study was supported by the Zhejiang Provincial Science \& Technology Foundation (grant no. 2014C33252).

\section{References}

1. Kolligs FT: Diagnostics and epidemiology of colorectal cancer. Visc Med 32: 158-164, 2016.

2. Williams R, White P, Nieto J, Vieira D, Francois F and Hamilton F: Colorectal cancer in African Americans: An update. Clin Transl Gastroenterol 7: e185, 2016.

3. Colman RJ and Rubin DT: Histological inflammation increases the risk of colorectal neoplasia in ulcerative colitis: A systematic review. Intest Res 14: 202-210, 2016.

4. He EY, Wyld L, Sloane MA, Canfell K and Ward RL: The molecular characteristics of colonic neoplasms in serrated polyposis: A systematic review and meta-analysis. J Pathol Clin Res 2: 127-137, 2016.

5. Okamoto K, Kitamura S, Kimura T, Nakagawa T, Sogabe M, Miyamoto H, Muguruma N and Takayama T: Clinicopathological characteristics of serrated polyps as precursors to colorectal cancer: Current status and management. J Gastroenterol Hepatol 32: 358-367, 2017.

6. De Craene B and Berx G: Regulatory networks defining EMT during cancer initiation and progression. Nat Rev Cancer 13: 97-110, 2013.

7. Shi M, Huang W, Lin L, Zheng D, Zuo Q, Wang L, Wang N, Wu Y, Liao Y and Liao W: Silencing of XB130 is associated with both the prognosis and chemosensitivity of gastric cancer. PLoS One 7: e41660, 2012.

8. Lee JY and Kong G: Roles and epigenetic regulation of epithelial-mesenchymal transition and its transcription factors in cancer initiation and progression. Cell Mol Life Sci 73: 4643-4660, 2016.

9. Smith BN and Bhowmick NA: Role of EMT in metastasis and therapy resistance. J Clin Med 5: pii: E17, 2016.

10. Liu X, Cai X, Hu B, Mei Z, Zhang D, Ouyang G, Wang J, Zhang $\mathrm{W}$ and Xiao W: Forkhead transcription factor $3 \mathrm{a}$ (FOXO3a) modulates hypoxia signaling via Up-regulation of the von Hippel-Lindau Gene (VHL). J Biol Chem 291: 25692-25705, 2016.

11. Shiozaki A, Shen-Tu G, Bai X, Iitaka D, De Falco V, Santoro M, Keshavjee S and Liu M: XB130 mediates cancer cell proliferation and survival through multiple signaling events downstream of Akt. PLoS One 7: e43646, 2012.

12. Zhou Y, Liang C, Xue F, Chen W, Zhi X, Feng X, Bai X and Liang T: Salinomycin decreases doxorubicin resistance in hepatocellular carcinoma cells by inhibiting the $\beta$-catenin/TCF complex association via FOXO3a activation. Oncotarget 6: 10350-10365, 2015.

13. Bai XH, Cho HR, Moodley S and Liu M: XB130-A novel adaptor protein: Gene, function and roles in tumorigenesis. Scientifica (Cairo) 2014: 903014, 2014

14. Xu J, Bai XH, Lodyga M, Han B, Xiao H, Keshavjee S, Hu J, Zhang H, Yang BB and Liu M: XB130, a novel adaptor protein for signal transduction. J Biol Chem 282: 16401-16412, 2007.

15. Wang X, Wang R, Liu Z, Hao F, Huang H and Guo W: XB130 expression in human osteosarcoma: A clinical and experimental study. Int J Clin Exp Pathol 8: 2565-2573, 2015.

16. Li J, Sun W, Wei H, Wang X, Li H and Yi Z: Expression of XB130 in human ductal breast cancer. Int J Clin Exp Pathol 8: 5300-5308, 2015.

17. Shi M, Zheng D, Sun L, Wang L, Lin L, Wu Y, Zhou M, Liao W, Liao Y, Zuo Q and Liao W: XB130 promotes proliferation and invasion of gastric cancer cells. J Transl Med 12: 1, 2014.

18. Jin C, Peng X, Liu F, Cheng L, Xie T, Lu X, Wu H and Wu N: Interferon-induced sterile alpha motif and histidine/aspartic acid domain-containing protein 1 expression in astrocytes and microglia is mediated by miR-181a. AIDS 30: 2053-2064, 2016. 
19. Jin C, Peng X, Liu F, Cheng L, Lu X, Yao H, Wu H and Wu N: MicroRNA-181 expression regulates specific post-transcriptional level of SAMHD1 expression in vitro. Biochem Biophys Res Commun 452: 760-767, 2014

20. Livak KJ and Schmittgen TD: Analysis of relative gene expression data using real-time quantitative PCR and the 2(-Delta Delta C(T)) method. Methods 25: 402-408, 2001.

21. de Vries NL, Swets M, Vahrmeijer AL, Hokland M and Kuppen PJ: The immunogenicity of colorectal cancer in relation to tumor development and treatment. Int J Mol Sci 17: pii: E1030, 2016
22. Gibson JA and Odze RD: Pathology of premalignant colorectal neoplasia. Dig Endosc 28: 312-323, 2016.

23. Zhang R, Zhang J, Wu Q, Meng F and Liu C: XB130: A nove adaptor protein in cancer signal transduction. Biomed Rep 4: 300-306, 2016.

24. Liu H, Yin J, Wang H, Jiang G, Deng M, Zhang G, Bu X, Cai S, $\mathrm{Du} \mathrm{J}$ and He Z: FOXO3a modulates WNT/ $\beta$-catenin signaling and suppresses epithelial-to-mesenchymal transition in prostate cancer cells. Cell Signal 27: 510-518, 2015. 